\title{
MicroRNA-106a promotes cell migration and invasion by targeting tissue inhibitor of matrix metalloproteinase 2 in cervical cancer
}

\author{
XIA LI* ${ }^{*}$ QI ZHOU, LING TAO* and CHUNXIA YU \\ Department of Obstetrics and Gynecology, The Affiliated Tumor Hospital \\ of Xinjiang Medical University, Urumqi, Xinjiang 830011, P.R. China
}

Received December 29, 2016; Accepted June 28, 2017

DOI: 10.3892/or.2017.5832

\begin{abstract}
Increasing evidence has demonstrated that miRNAs play a critical role in tumor development and progression. Previous studies have revealed that miR-106a is abnormally expressed in various cancers. However, its function and underlying mechanism in cervical cancer (CC) remains unknown. In this study, we confirmed that the expression of miR-106a was significantly upregulated in both CC cell lines and tissues by qRT-PCR. The increased expression of miR-106a was obviously associated with adverse prognostic features. Moreover, we demonstrated that miR-106a was a novel independent prognostic marker for predicting the 5-year survival of CC patients. The ectopic overexpression of miR-106a promoted cell migration, invasion and invasion-related gene expression, while downregulated miR-106a reversed the effect. In addition, miR-106a regulated tissue inhibitor of metalloproteinase (TIMP)2 by directly binding to its 3'-UTR, leading to the indution of the expression of matrix metalloproteinases (MMPs). In clinical samples of CC, miR-106a was inversely correlated with TIMP2, which was downregulated in CC. Alteration of TIMP2 expression at least partially abolished the migration, invasion and MMP expression of miR-106a in CC cells. In conclusion, our data indicated that miR-106a promoted the migration, invasion and MMP expression of CC by targeting TIMP2, and may represent a novel potential therapeutic target and prognostic marker for $\mathrm{CC}$.
\end{abstract}

\section{Introduction}

Cervical cancer (CC) is one of the most common cancers and the third leading cause of cancer-related deaths in women

Correspondence to: Professor Qi Zhou, Department of Obstetrics and Gynecology, The Affiliated Tumor Hospital of Xinjiang Medical University, 789 Suzhou East Urumqi Road, Urumqi, Xinjiang 830011, P.R. China

E-mail: zhouq0615@126.com

${ }^{*}$ Contributed equally

Key words: microRNA-106a, cervical cancer, TIMP2, MMP, invasion worldwide $(1,2)$. Despite notable advances in surgery, radiotherapy and chemotherapy, even the novel molecular-targeted therapy, the long-term survival of advanced CC patients remains poor due to the high rates of tumor recurrence and distal metastasis (3-5). To elucidate the development and progression of $\mathrm{CC}$, it is crucial to identify the molecular etiology and molecular mechanisms underlying the progression and metastasis in $\mathrm{CC}$ and thus improve therapeutic strategies and prognosis.

MicroRNAs (miRNAs) are a class of small, non-coding RNAs that negatively regulate protein expression by binding to the 3'-untranslated region (3'-UTR) of target mRNAs and function as post-transcriptional regulators of gene expression $(6,7)$. Accumulating studies have confirmed that aberrant expression of miRNAs is involved in various types of cancer, including $\mathrm{CC}$, and participate in diverse biological processes such as cell growth, invasion, differentiation and metastasis (8-12). Recently, miR-106a, which is derived from the precursor miR-106a-363 on chromosome Xq26.2, was revealed to play a critical role in the progression of cancers (13-17). A previous study revealed that miR-106a functions as a regulator of cell invasion, proliferation and drug sensitivity (18). miR-106a functioned as an oncogene in human gastric cancer and contributed to proliferation and metastasis in vitro and in vivo and a high expression of miR-106a was associated with the poor survival rate of gastric cancer patients $(19,20)$. Upregulated expression of miR-106a by DNA hypomethylation played an oncogenic role in hepatocellular carcinoma by targeting TP53INP1 and CDKN1A (21). However, the expression of miR-106a was downregulated in glioma, renal carcinoma and osteosarcoma cancer (22-24). miR-106a inhibited glioma cell growth by targeting E2F1 independent of p53 status (25). miR-106a inhibited the proliferation of renal carcinoma cells by targeting IRS-2 (24). Moreover, miR-106a suppressed the proliferation, migration and invasion of osteosarcoma cells by targeting HMGA2 (22). Thus, the functional significance of miR-106a in cancer initiation, development and progression appears to be cancer-type specific. However, the functional role and the underlying molecular mechanism by which miR-106a regulates the development and progression of CC have not been elucidated. 
In this study, we investigated the expression and biological function of miR-106a in CC progression. We demonstrated that the expression of miR-106a was upregulated in both $\mathrm{CC}$ tissues and cell lines. Increased expression of miR-106a was associated with adverse prognostic characteristics and poor 5-year survival of CC patients. MiR-106a promoted CC migration and invasion in vitro by gain- and loss-of-function experiments by directly targeting tissue inhibitor of metalloproteinase (TIMP)2. Collectively, these data confirmed the underlying mechanism by which miR-106a promoted migration and invasion of CC and identified miR-106a as a novel prognostic biomarker for $\mathrm{CC}$ patients.

\section{Materials and methods}

Clinical samples and cell culture. CC tissues and corresponding adjacent normal tissues were obtained from 135 patients who received routine surgery at the Affiliated Tumor Hospital of Xinjiang Medical University from January 2007 to December 2010. None of the patients had received any therapy before surgery. All tissues were stored at $-80^{\circ} \mathrm{C}$ until RNA extraction. Informed consent was obtained from all the patients and this study was approved by the ethics committee of the local institutional review boards in accordance with the Declaration of Helsinki.

Four CC cell lines (C33A, HeLa, CaSki, SiHa) and a normal human cervical epithelial cell line (H8) were obtained from the Institute of Biochemistry and Cell Biology (Chinese Academy of Sciences, Shanghai, China) and were cultured in Dulbecco's modified Eagle's medium (DMEM; Hyclone, Logan, UT, USA) containing 10\% fetal bovine serum (FBS; Gibco, Grand Island, NY, USA) with $1 \%$ penicillin /streptomycin (Sigma, St. Louis, MO, USA) at $37^{\circ} \mathrm{C}$ with $5 \% \mathrm{CO}_{2}$.

Quantitative real-time reverse transcription polymerase chain reaction $(q R T-P C R)$. TRIzol reagent (Invitrogen, Carlsbad, CA, USA) was used to extract RNA from tissues and cell lines according to the manufacturer's instructions. RNA was reverse-transcribed using a TaqMan human miRNA assay kit (Applied Biosystems, Foster City, CA, USA) and cDNA was then amplified with a SYBR ${ }^{\circledR}$ Premix Ex Taq ${ }^{\mathrm{TM}}$ II (Perfect Real-Time) kit (Takara Bio, Inc., Shiga, Japan) and analyzed with an Applied Biosystems 7900 real-time PCR system. Hsa-miR-106a primer (HmiRQP0027), snRNA U6 qPCR primer (HmiRQP9001) and GAPDH (HQP006940) were purchased from Genecopoeia (Guangzhou, China). The primers for TIMP2 were as follows: sense, 5'-AGCACCACCC AGAAGAAGAG-3'; and antisense, 5'-GTGACCCAGTCCAT CCAGAG-3' and were synthesized by GenePharma (Shanghai, China).

Western blot analysis. Whole tissue and cell proteins were collected in RIPA buffer (Beyotime, China) and the protein concentration was determined using a BCA protein assay kit (Thermo Scientific, Rockford, IL, USA). An equal amount of protein was separated by $10 \%$ SDS-PAGE and transferred to PVDF membranes (Millipore, Billerica, MA, USA). The membranes were blocked with $5 \%$ non-fat milk in TBST and then incubated with primary antibodies: TIMP2, matrix metalloproteinase (MMP)2, MMP9 and GAPDH (1:1,000;
Cell Signaling Technology, Inc.) at $4^{\circ} \mathrm{C}$ overnight. After being washed by TBST, the membranes were incubated with an appropriate peroxidase-conjugated secondary antibody for $2 \mathrm{~h}$ at room temperature (ZSGB-Bio, China). The protein bands were visualized using an enhanced chemiluminescence kit (Amersham, Little Chalfont, UK).

Immunohistochemical analysis (IHC). IHC was performed on 4- $\mu \mathrm{m}$ thickness of paraformaldehyde-fixed paraffin sections. MMP2 and MMP9 (1:100; Cell Signaling Technology, Inc., Danvers, MA, USA) antibodies were used in immunohistochemistry with the streptavidin peroxidase-conjugated (SP-IHC) method. The staining results for the protein were semiquantitatively calculated by multiplying the staining intensity and the percentage of positive cells. The staining intensity was expressed as four grades: $0=$ none; $1=$ weak; $2=$ moderate ; and $3=$ strong. The percentage of positive cells was expressed as the following grades: $0,<5 \% ; 1,6-25 \% ; 2$, 26-50\%; 3, 51-75\%; and 4, $>75 \%$.

Cell transfection. The cells were seeded in 6-well plates and transfected with vectors using Lipofectamine 2000 reagent (Invitrogen Life Technologies) in accordance with the manufacturer's protocol. miRNA expression vectors, including miR-106a expression vector (HmiR0392), the control vector (CmiR0001-MR04), the miR-106a inhibitor (HmiR-AN0027) and the negative control (CmiR-AN0001-AM04) were obtained from Genecopoeia. The TIMP2 expression plasmid and specific siRNA were producted by GenePharma.

Transwell migration and invasion assays. Cell migration and invasion assays were assessed by $8-\mu \mathrm{M}$ pore-sized Transwell inserts (Nalge Nunc International Corp., Naperville, IL, USA). Cells $\left(2.5 \times 10^{4}\right)$ in $200 \mu \mathrm{l}$ of serum-free DMEM medium and were loaded into the top chamber, and $750 \mu 1$ of DMEM medium with $10 \%$ FBS were placed in the bottom chamber. For the invasion, the upper chambers were pre-coated with Matrigel (BD Biosciences). After $24 \mathrm{~h}$ of incubation, the cells were fixed in $4 \%$ paraformaldehyde and stained with $0.3 \%$ crystal violet. The cells remaining in the top layer were swabbed carefully and the bottom cells were counted under a microscope.

Luciferase reporter assay. Luciferase activity was determined by the Dual-Luciferase assay (Promega, Madison, WI, USA) according to the manufacturer's protocol. The wild-type 3'-UTR of TIMP2 containing the putative miR-106a target site was amplified and cloned into the pGL3-control luciferase reporter vector (Promega). The mutant constructs were generated using a QuickChange Site-Directed Mutagenesis kit (Stratagene, La Jolla, CA, USA). The experiment was performed in duplicate in three independent experiments.

Statistical analysis. Data are presented as the mean \pm SD from at least three independent replicates. SPSS software, 16.0 (SPSS., Inc, Chicago, IL, USA) and Graphpad Prism 6.0 (GraphPad Software, Inc., La Jolla, CA, USA) were used for a two-tailed Student's t-test, Pearson's correlation analysis, Kaplan-Meier method and the log-rank test to evaluate the statistical significance. Differences were defined as $\mathrm{P}<0.05$. 
A

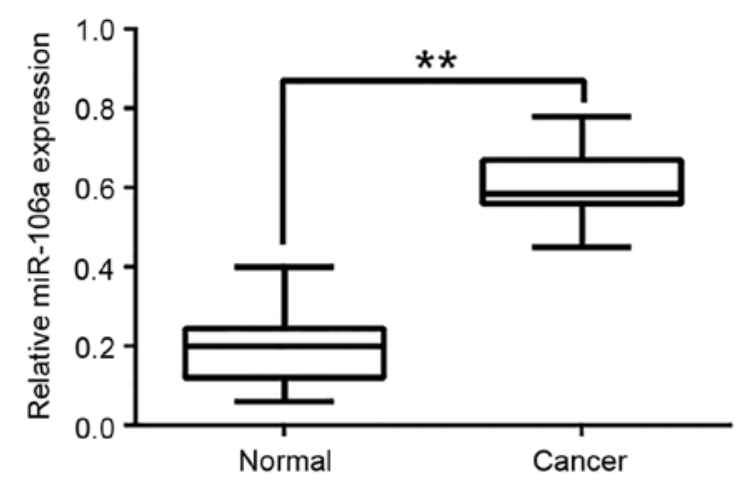

B

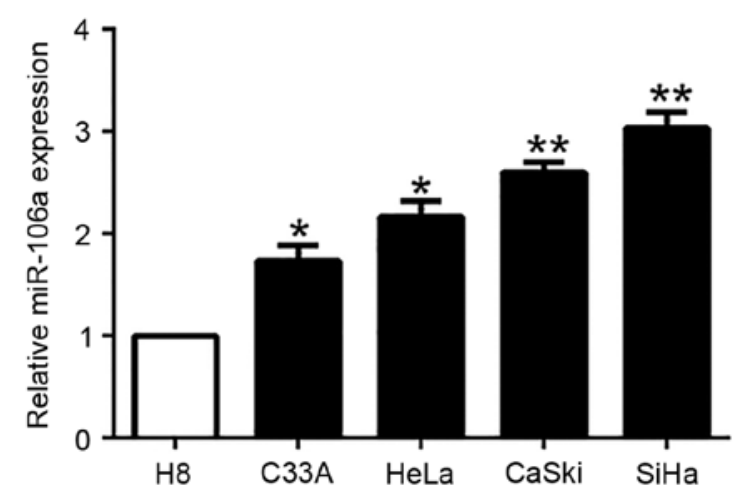

Figure 1. Expression of miR-106a in CC tissues and cell lines. (A) Quantification of the data revealed that the mean level of miR-106a expression in CC tissues was significantly higher than that in matched adjacent non-cancer tissues. (B) Differences in the expression levels of miR-106a between $\mathrm{CC}$ cell lines compared to the normal human cervical epithelial cell line (H8). $n=6$ repeats with similar results. U6 snRNA was used as an internal control. ${ }^{*} \mathrm{P}<0.05,{ }^{* *} \mathrm{P}<0.01$. CC, cervical cancer.

\section{Results}

miR-106a is significantly upregulated in CC tissues and cell lines. To explore the expression of miR-106a in CC tissues and cell lines, qRT-PCR was performed. We found that miR-106a expression was significantly higher in human $\mathrm{CC}$ tissues than that of adjacent normal cervical tissues $(\mathrm{P}<0.05$, Fig. 1A). Furthermore, we analyzed miR-106a expression in a panel of CC cell lines and normal human cervical epithelial cell line (H8). Consistently, our data revealed that miR-106a was significantly upregulated in the $\mathrm{CC}$ cell lines compared to the $\mathrm{H} 8$ cells $(\mathrm{P}<0.05$, Fig. $1 \mathrm{~B})$. These results indicated that the increased expression of miR-106a is potentially correlated with the development and progression of CC.

Correlation between the expression of miR-106a and the clinical characteristics in CC samples. To assess its clinical relevance in CC samples, we defined the high and low expression of miR-106a according to the median expression level. As shown in Table I, high miR-106a expression was significantly associated with lymph node metastasis (LNM, P=0.007), lymphovascular space invasion (LVSI, $\mathrm{P}=0.001$ ) and advanced FIGO stage $(\mathrm{P}=0.038)$. Hence, these findings indicated that increased miR-106a was involved in the development and progression of CC. Moreover, survival analysis revealed that
Table I. Clinical correlation of miR-106a expression in CC $(n=135)$.

\begin{tabular}{|c|c|c|c|c|}
\hline \multirow[b]{2}{*}{$\begin{array}{l}\text { Clinical } \\
\text { parameters }\end{array}$} & \multirow[b]{2}{*}{$\begin{array}{l}\text { Cases } \\
\text { (n) }\end{array}$} & \multicolumn{2}{|c|}{ Expression level } & \multirow[b]{2}{*}{$\begin{array}{l}\text { P-value } \\
\left({ }^{\mathrm{a}} \mathrm{p}<0.05\right.\end{array}$} \\
\hline & & $\begin{array}{l}\operatorname{miR}-106 a^{\text {high }} \\
\quad(n=73)\end{array}$ & $\begin{array}{c}\mathrm{miR}-106 \mathrm{a}^{\text {low }} \\
(\mathrm{n}=62)\end{array}$ & \\
\hline Age (years) & & & & 0.642 \\
\hline$<45$ & 33 & 19 & 14 & \\
\hline$\geq 45$ & 102 & 54 & 48 & \\
\hline FIGO stage & & & & $0.038^{\mathrm{a}}$ \\
\hline I & 107 & 53 & 54 & \\
\hline II & 28 & 20 & 8 & \\
\hline Tumor size $(\mathrm{cm})$ & & & & 0.680 \\
\hline$<4$ & 109 & 58 & 51 & \\
\hline$\geq 4$ & 26 & 15 & 11 & \\
\hline LNM & & & & $0.007^{\mathrm{a}}$ \\
\hline Negative & 117 & 58 & 59 & \\
\hline Positive & 18 & 15 & 3 & \\
\hline LVSI & & & & $0.001^{\mathrm{a}}$ \\
\hline Negative & 112 & 53 & 59 & \\
\hline Positive & 23 & 20 & 3 & \\
\hline Vaginal invasion & & & & 0.992 \\
\hline Negative & 111 & 60 & 51 & \\
\hline Positive & 24 & 13 & 11 & \\
\hline Histology & & & & 0.625 \\
\hline Squamous & 120 & 64 & 56 & \\
\hline Adenocarcinoma & 15 & 9 & 6 & \\
\hline $\begin{array}{l}\text { Parametrial } \\
\text { extension }\end{array}$ & & & & 0.570 \\
\hline Negative & 122 & 65 & 57 & \\
\hline Positive & 13 & 8 & 5 & \\
\hline
\end{tabular}

CC, cervical cancer; FIGO, International Federation of Gynecology and Obstetrics; LNM, lymph node metastasis; LVSI, lymphovascular space invasion. ${ }^{\mathrm{a}} \mathrm{P}<0.05$.

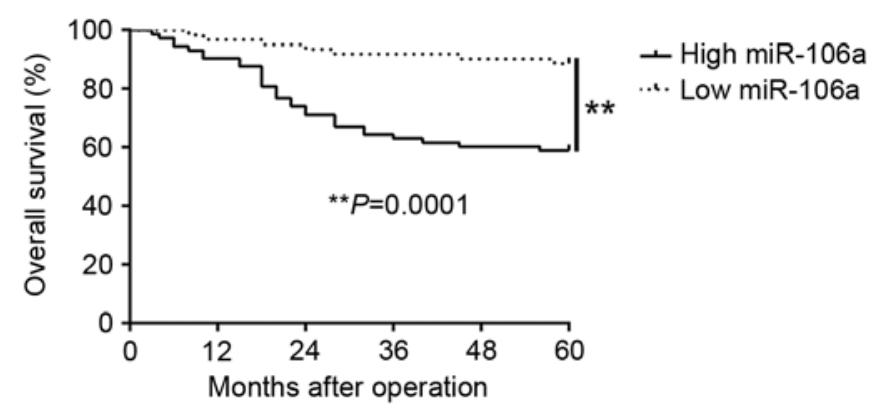

Figure 2. The prognostic value of miR-106a for CC patients. CC patients with higher expression of miR-106a had a poorer overall survival. ${ }^{* *} \mathrm{P}<0.01$. CC, cervical cancer.

upregulation of miR-106a was markedly correlated with shorter overall survival ( $\mathrm{P}=0.0001$, Fig. 2 ) in $\mathrm{CC}$ patients. Furthermore, miR-106a expression was an independent prognostic factor for predicting the overall survival in CC patients 
Table II. Univariate and multivariate analyses of the 5-year overall survival of $135 \mathrm{CC}$ patients.

\begin{tabular}{|c|c|c|c|c|c|c|}
\hline \multirow[b]{2}{*}{ Variables } & \multicolumn{3}{|c|}{ Univariate analysis } & \multicolumn{3}{|c|}{ Multivariate analysis } \\
\hline & HR & $95 \% \mathrm{CI}$ & $\mathrm{P}$-value & HR & $95 \% \mathrm{CI}$ & P-value \\
\hline miR-106a expression & 4.832 & $1.852-12.257$ & $0.002^{\mathrm{a}}$ & 3.982 & $1.382-10.237$ & $0.009^{\mathrm{a}}$ \\
\hline FIGO stage & 1.543 & $1.083-5.985$ & $0.032^{\mathrm{a}}$ & 1.123 & $1.108-3.668$ & $0.038^{\mathrm{a}}$ \\
\hline LNM & 3.192 & $1.426-7.678$ & $0.007^{\mathrm{a}}$ & 1.747 & $1.212-4.649$ & $0.018^{\mathrm{a}}$ \\
\hline LVSI & 3.312 & $1.382-7.681$ & $0.014^{\mathrm{a}}$ & 1.223 & $1.019-5.348$ & $0.023^{\mathrm{a}}$ \\
\hline
\end{tabular}

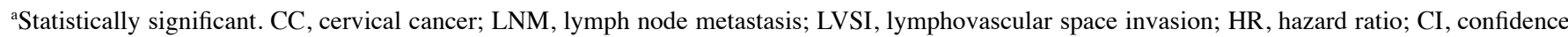
interval.
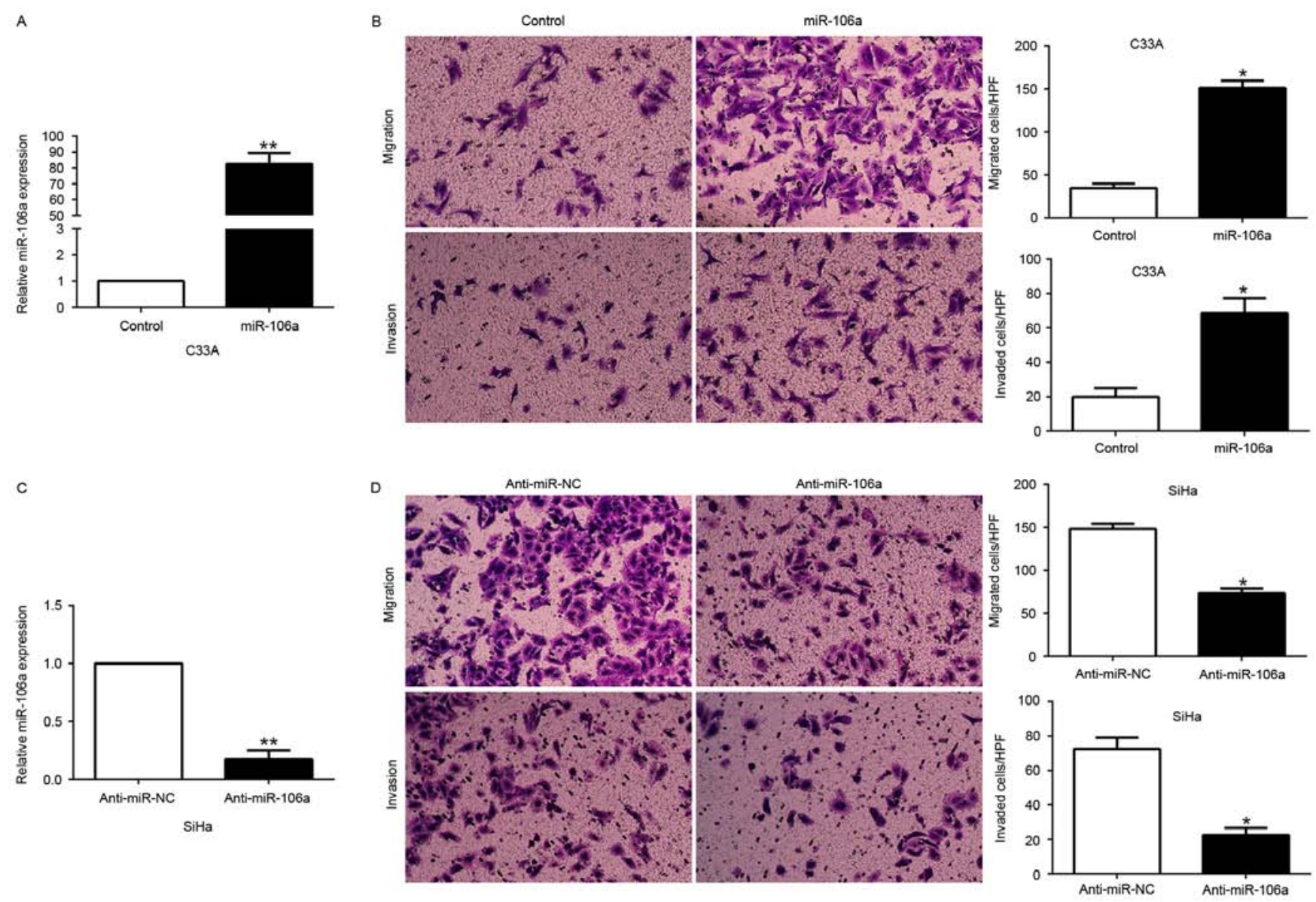

Figure 3. miR-106a promotes CC cell migration and invasion in vitro. (A) C33A cells that were transfected with corresponding miRNA vectors were subjected to qRT-PCR to assess the expression of miR-106a. (B) Cell migration and invasion as assessed by Transwell assays were promoted by overexpression of miR-106a in C33A cells. (C) SiHa cells that were transfected with a miR-106a inhibitor (anti-miR-106a) and a negative control were subjected to qRT-PCR to determine the expression of miR-106a. (D) Cell migration and invasion as assessed by Transwell assays were inhibited by knockdown of miR-106a in SiHa cells. $\mathrm{n}=6$ independent experiments. ${ }^{*} \mathrm{P}<0.05,{ }^{* *} \mathrm{P}<0.01$. CC, cervical cancer.

( $\mathrm{P}=0.002,0.009$, respectively, Table II). These results revealed that miR-106a could serve as a potential prognostic biomarker in CC patients.

miR-106a promotes CC cell migration and invasion in vitro. To further illustrate the biological function of miR-106a in $\mathrm{CC}$ cells, we transfected the miR-106a expression vector into C33A cells or the anti-miR-106a vector into SiHa cells which contained different endogenous miR-106a levels. As assessed by qRT-PCR, we confirmed that miR-106a effectively increased
miR-106a in the C33A cells $(\mathrm{P}<0.05$, Fig. 3A) or decreased miR-106a in the SiHa cells $(\mathrm{P}<0.05$, Fig. $3 \mathrm{C})$. Transwell migration and invasion assays were performed to evaluate the effects of miR-106a on the migration and invasion of the $\mathrm{C} 33 \mathrm{~A}$ and $\mathrm{SiHa}$ cells. We found that miR-106a overexpression significantly promoted the migration and invasion of the C33A cells ( $\mathrm{P}<0.05$, Fig. 3B), whereas miR-106a knockdown markedly inhibited the number of migrated and invaded $\mathrm{SiHa}$ cells $(\mathrm{P}<0.05$, Fig. 3D). These data revealed that miR-106a promoted CC cell migration and invasion in vitro. 


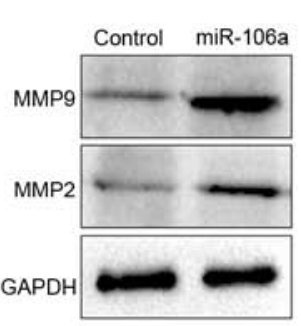

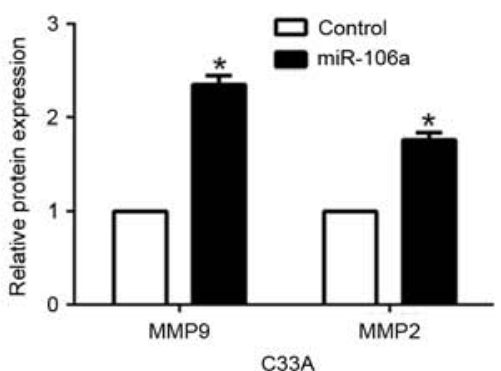

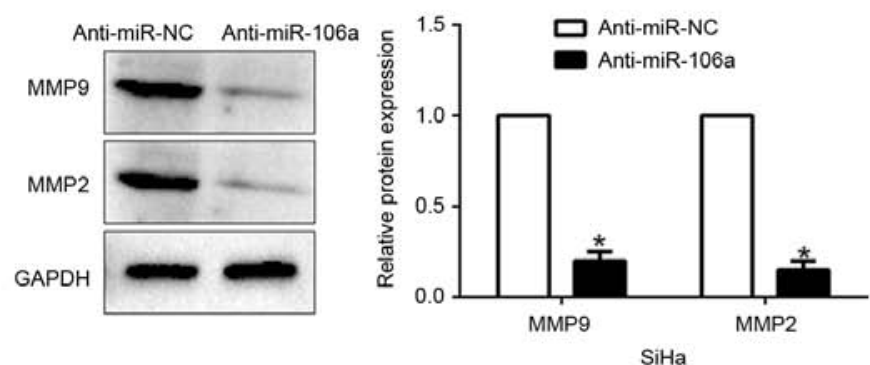

C
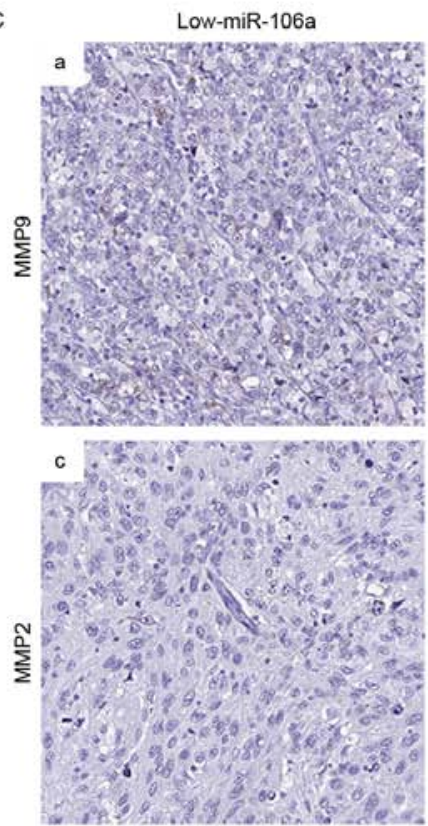
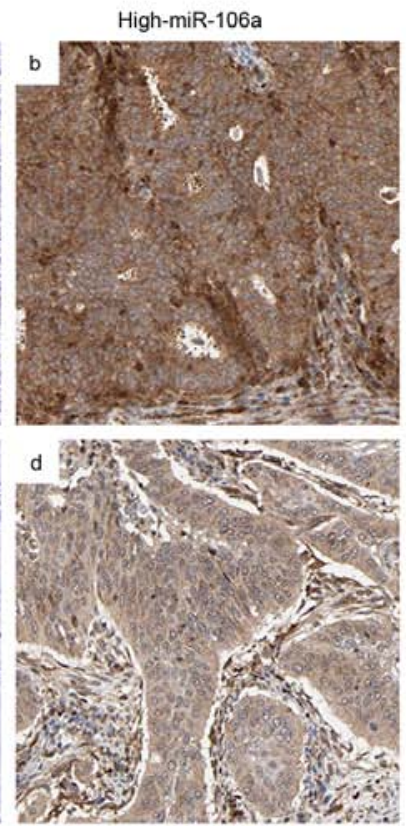
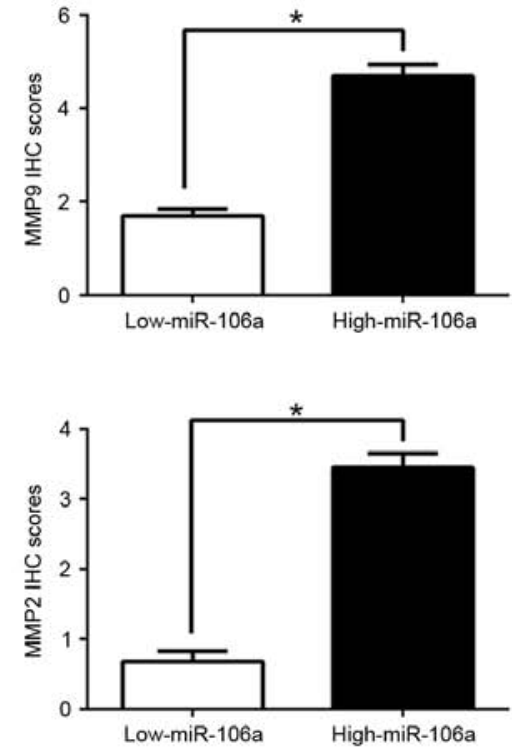

Figure 4. miR-106a promotes MMP2 and MMP9 expression in CC cells. (A) The overexpression of miR-106a in C33A cells increased the expression of MMP2 and MMP9. (B) Conversely, the expression of the antagomiR to miR-106a decreased the expression of MMP2 and MMP9. (C) Immunohistochemical analysis of MMP2 and MMP9 in CC samples. In cases of low miR-106a expression (a and c); there was no detectable MMP2 and MMP9 protein expression in the tissue sections. In contrast, in the case of high miR-106a expression (b and d), there was strong MMP2 and MMP9 protein expression. Values are depicted as the mean $\pm \mathrm{SEM} ;{ }^{*} \mathrm{P}<0.05$ by t-test. MMP, matrix metalloproteinase; $\mathrm{CC}$, cervical cancer.

miR-106a modulates the expression of MMPs. Previous studies confirmed that MMP2 and MMP9 are important members of the MMP family, which play critical roles in cancer migration and invasion, including CC. Thus, we investigated whether miR-106a could regulate invasion-related genes. Our data revealed that miR-106a overexpression significantly upregulated the expression of MMP2 and MMP9 (P<0.05, Fig. 4A), however, miR-106a knockdown markedly downregulated the expression of MMPs ( $\mathrm{P}<0.05$, Fig. 4B). In addition, we further explored the correlation between miR-106a expression and MMPs in CC tissues. We found that the expression of MMP2 and MMP9 in the high miR-106a-expression group was higher than that in the low miR-106a-expression group ( $\mathrm{P}<0.05$, Fig. $4 \mathrm{C})$. Collectively, these results revealed that miR-106a is vital for $\mathrm{CC}$ cell invasion by modulating the expression of MMPs.

TIMP2 is a direct target of miR-106a in CC cells. To investigate the mechanism of miR-106a in CC cell migration and invasion, we used algorithm TargetScan to search for candidate targets of miR-106a and found that the 3'-UTR of TIMP2 matched the 'seed sequence' of miR-106a (Fig. 5A). To ascertain whether TIMP2 was a direct target of miR-106a in $\mathrm{CC}$ cells, we carried out a luciferase reporter assay to confirm that miR-106a could bind to the 3'-UTR of TIMP2. The reporter assays revealed that the increased miR-106a markedly inhibited the luciferase activity of the wild-type (wt) TIMP2 3'-UTR $(\mathrm{P}<0.05$, Fig. 5B) while it had no influence on that of the mutant (mt) TIMP2 3'-UTR (P>0.05, Fig. 5B). Conversely, the miR-106a decrease increased the luciferase activity of the wt TIMP2 3'-UTR $(\mathrm{P}<0.05$, Fig. 5B) but did not affect the luciferase activity of the mt TIMP2 3'-UTR constructs. Furthermore, miR-106a overexpression markedly suppressed the mRNA and protein expression of TIMP2 in the C33A cells $(\mathrm{P}<0.05$, respectively, Fig. 5C and D). By contrast, the expression of TIMP2 mRNA and protein were significantly increased by the inhibition of miR-106a in the SiHa cells $(\mathrm{P}<0.05$, respectively, Fig. 5C and D).

miR-106a expression is inversely correlated with TIMP2 in $C C$ tissues. To further investigate the relationship between miR-106a and TIMP2 in vivo, we examined the mRNA and protein expression in diverse miR-106a expression groups. The results revealed that TIMP2 mRNA and protein levels were significantly lower in the high miR-106a-expression group than that in the low miR-106a-expression group in $\mathrm{CC}(\mathrm{P}<0.05$, Fig. 6A and $\mathrm{B})$. In addition, we demonstrated 
A

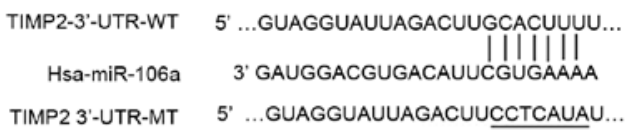

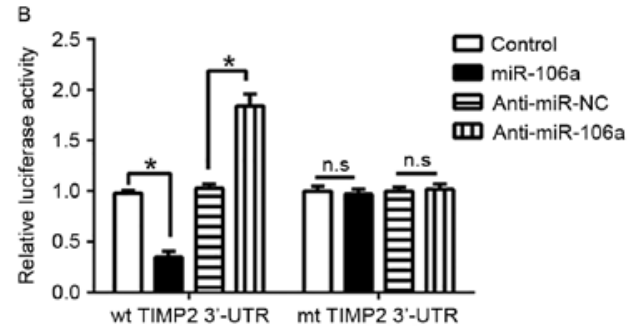
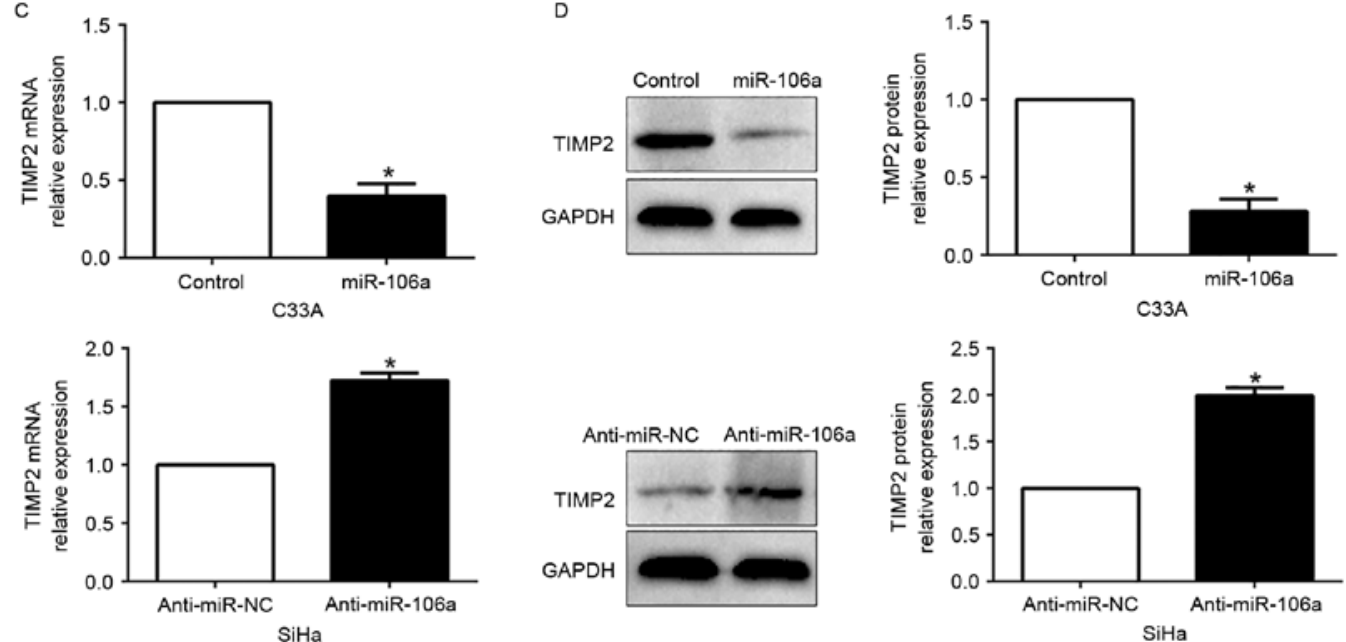

Figure 5. TIMP2 is identified as a direct target of miR-106a in CC. (A) miR-106a and its putative binding sequence in the 3'-UTR of TIMP2. The mutant binding site was generated in the complementary site for the seed region of miR-106a. (B) miR-106a significantly suppressed the luciferase activity that carried the wild-type (wt) but not the mutant (mt) 3'-UTR of TIMP2. Anti-miR-106a led to a notable increase in the luciferase activity of the wt 3'-UTR of TIMP2. (C) qRT-PCR analysis of TIMP2 mRNA expression in the C33A cells with miR-106a or miR-control vector transfection and the SiHa cells with anti-miR-106a or anti-miR-NC vector transfection. (D) Overexpression of miR-106a decreased the expression of the TIMP2 protein in the C33A cells and knockdown of miR-106a increased the level of the TIMP2 protein in the SiHa cells. $\mathrm{n}=6$ repeats with similar results, ${ }^{*} \mathrm{P}<0.05$. TIMP, tissue inhibitors of metalloproteinase; CC, cervical cancer; 3'-UTR, 3'-untranslated region.

A

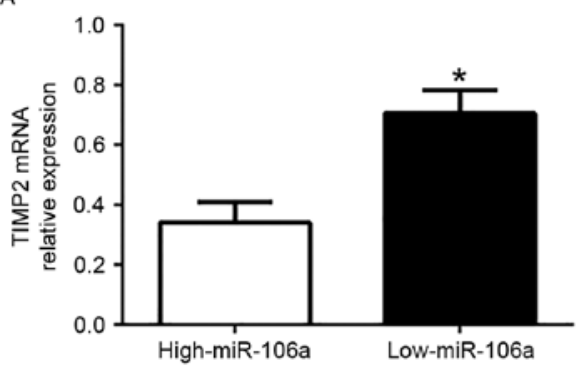

B

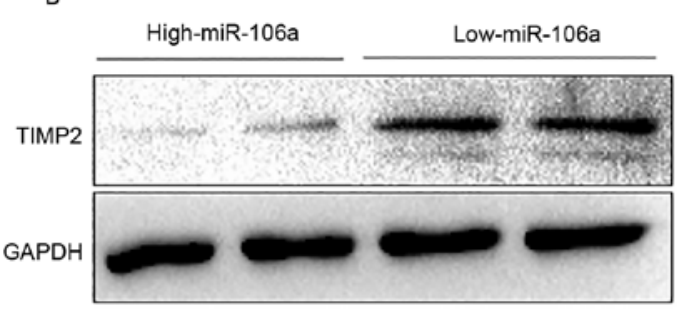

C
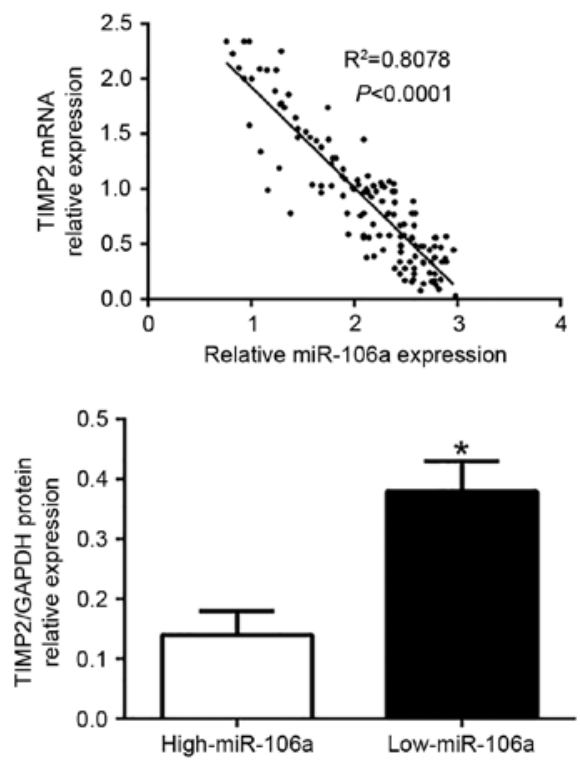

Figure 6. An inverse correlation between miR-106a and TIMP2 expression is observed in CC. CC tissues (135) were involved. (A) The expression of TIMP2 mRNA in the miR-106a high-expressing tumors was significantly lower than that in the miR-106a low-expressing tumors. (B) The expression of TIMP2 protein in the miR-106a high-expressing tumors was significantly lower than that in the miR-106a low-expressing tumors. (C) A significant inverse correlation between the mRNA levels of TIMP2 and miR-106a was observed in CC tissues. ${ }^{*} \mathrm{P}<0.05$. TIMP, tissue inhibitors of metalloproteinase; CC, cervical cancer.

that the mRNA level of TIMP2 in the CC tissues was inversely correlated with miR-106a expression $(\mathrm{R} 2=0.8078$,
$\mathrm{P}<0.0001$, Fig. 6C). In conclusion, these data revealed that TIMP2 was a direct downstream target of miR-106a in CC. 


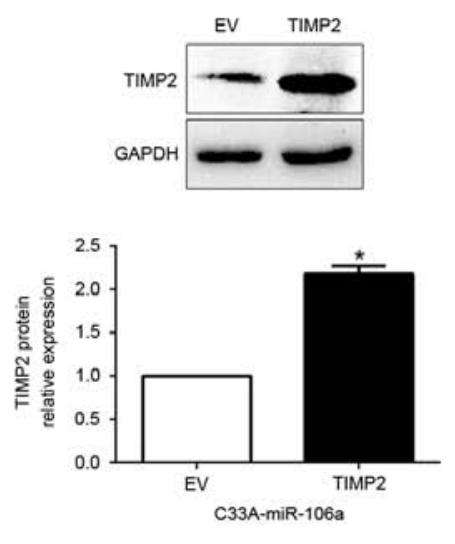

C

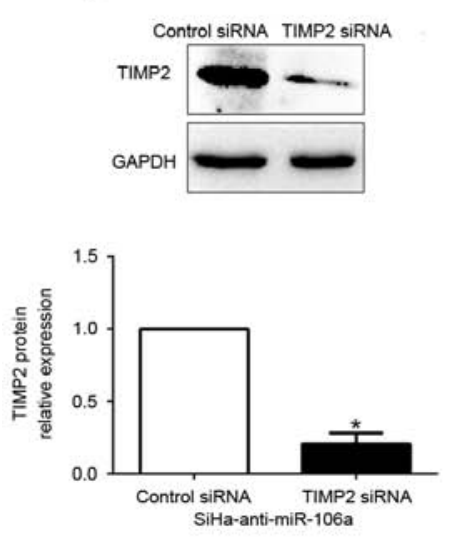

E

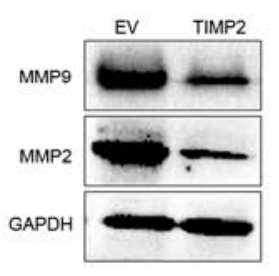

B
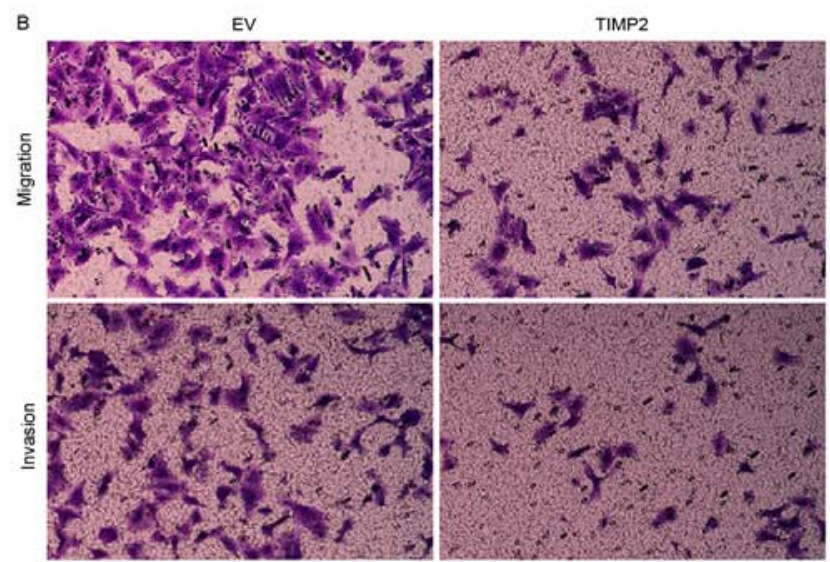

D
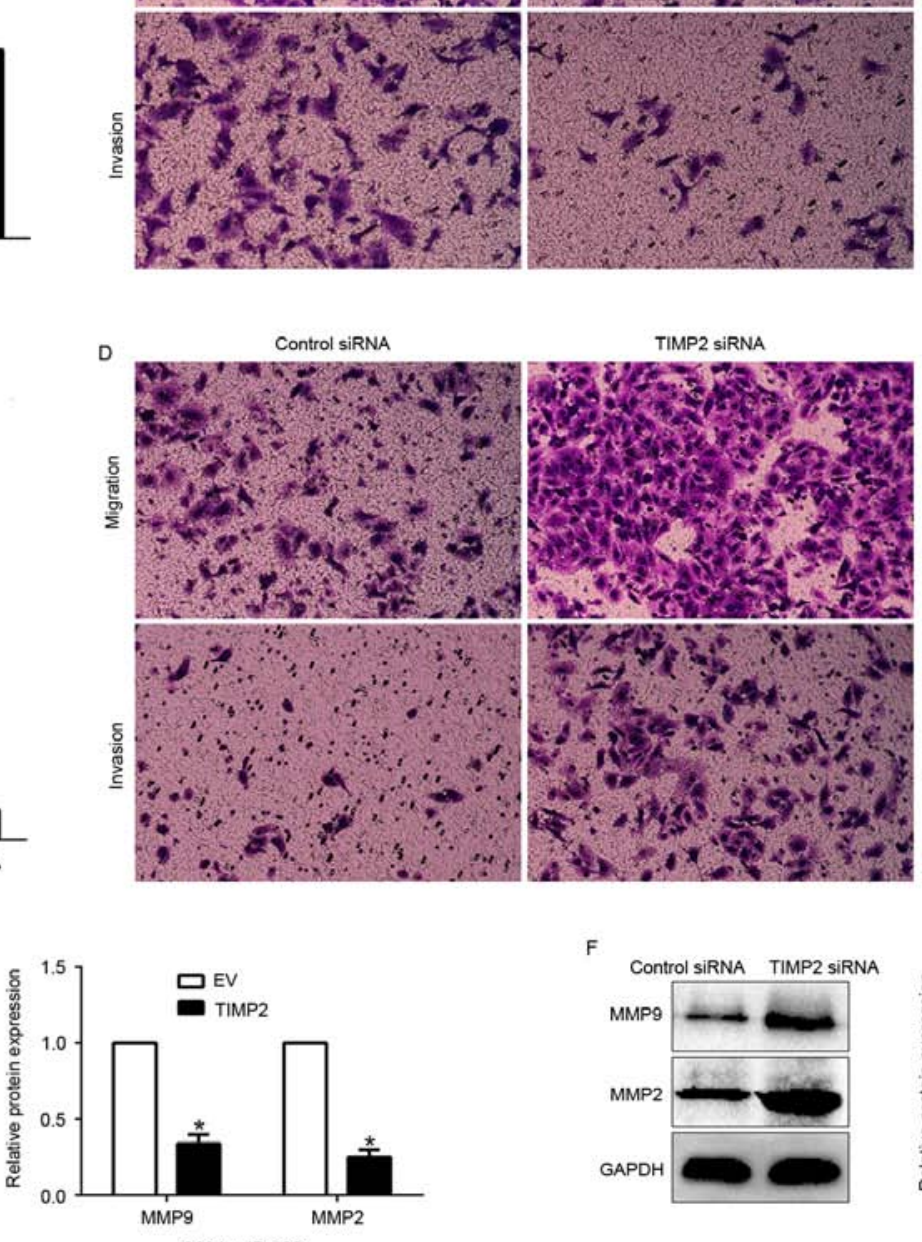

TIMP2 SIRNA

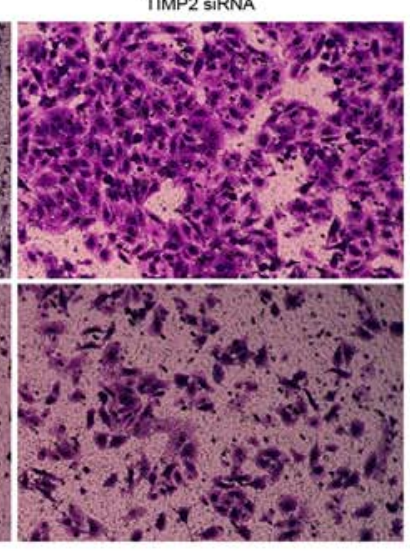

F

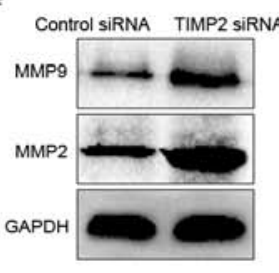

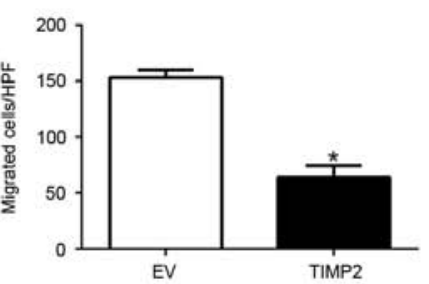
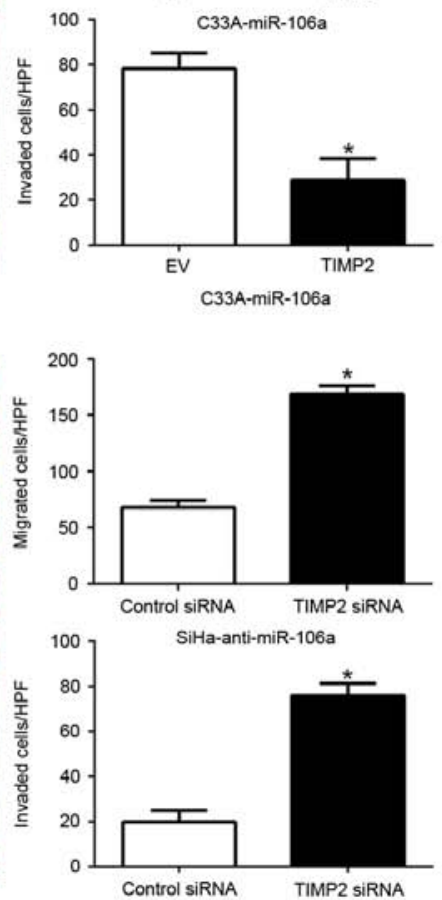

SiHa-anti-miR-106a

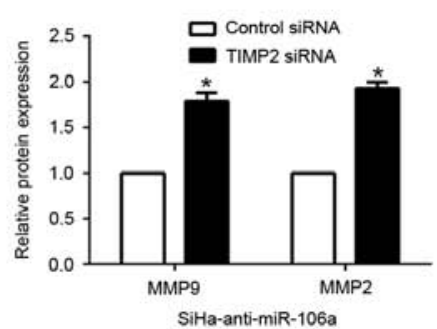

Figure 7. Alterations of TIMP2 partially abolish miR-106a-mediated CC cell migration, invasion and the expression of MMPs. (A) miR-106a-overexpressing C33A cells that were transfected with EV or TIMP2 expression plasmid were subjected to western blot analysis for TIMP2. (B) The cell migration and invasion of the miR-106a-overexpressing C33A cells was inhibited by TIMP2 overexpression. (C) miR-106a-suppressive SiHa cells that were transfected with scrambled siRNA or TIMP2-siRNA were subjected to western blot analysis for TIMP2. (D) TIMP2 knockdown abrogated the effects of miR-106a knockdown on SiHa cells. (E) Western blot analysis of MMP2 and MMP9 expression in C33A cells stably expressing miR-106a transduced with TIMP2 or control vector. (F) Western blot analysis of indicated proteins in SiHa cells stably expressing the miR-106a inhibitor transfected with TIMP2-siRNA or control siRNA. $\mathrm{n}=6$ independent experiments. EV, empty vector. ${ }^{*} \mathrm{P}<0.05$. TIMP, tissue inhibitors of metalloproteinase; CC, cervical cancer; MMP, matrix metalloproteinase.

Functional role of TIMP2 in miR-106a-mediated cell migration, invasion and invasion-related MMP expression. Having confirmed TIMP2 as a target of miR-106a, we next explored the biological function of TIMP2 in miR-106a-mediated cell migration, invasion and increase of MMPs. We restored TIMP2 expression in the C33A-miR-106a cells by transfecting them with TIMP2 expression plasmid ( $\mathrm{P}<0.05$, Fig. 7A). Furthermore, TIMP2 overexpression inhibited the cell migration, and invasion $(\mathrm{P}<0.05$, respectively, Fig. 7B) and suppressed the expression of MMPs ( $\mathrm{P}<0.05$, Fig. 7E). Conversely, TIMP2 knockdown by a specific siRNA in the miR-106a-suppressive SiHa cells $(\mathrm{P}<0.05$, Fig. 7C) significantly increased cell migration and invasion $(\mathrm{P}<0.05$, respectively, Fig. 7D) and promoted the expression of MMPs $(\mathrm{P}<0.05$, Fig. 7F). These data demonstrated that TIMP2 is a downstream mediator in the function of miR-106a in CC.

\section{Discussion}

Accumulating evidence has demonstrated that aberrant expression of miRNAs plays pivotal roles in the development and progression of cancer by inhibiting the expression of their target genes and potentially serve as biomarkers for the prediction and prognosis in diverse cancers, including CC (26-28). In previous studies, Zhu et al demonstrated that miR-106a targets TIMP2 to regulate the invasion and metastasis of gastric cancer (15). The phenomenon was also 
observed in pancreatic cancer (16). These data strongly support our present data in regards to $\mathrm{CC}$. Moreover, miR-106a was frequently upregulated in gastric cancer and inhibited the extrinsic apoptotic pathway by targeting FAS (13). In addition, miR-106a targeted autophagy and enhanced sensitivity of lung cancer cells to Src inhibitors (29). However, conversely, miR-106a inhibited the proliferation and migration of astrocytoma cells and promoted apoptosis by targeting FASTK (23). Moreover, miR-106a suppressed the proliferation, migration, and invasion of osteosarcoma cells by targeting HMGA2 (22). These data indicated that the expression level and biological effect of miR-106a was dependent on the type of cancer.

In present study, we found that miR-106a was significantly upregulated in CC tissue samples and cell lines, which was consistent with previous microarray analyses data (30). Increased miR-106a expression was prominently associated with malignant clinicopathological features of $\mathrm{CC}$ patients, including lymph node metastasis, lymphovascular space invasion and advanced FIGO stage. Moreover, we found that the high miR-106a-expression group had a poorer 5-year overall survival for CC patients than the low miR-106a-expression group. Multivariate Cox regression analysis revealed that miR-106a was an independent prognostic factor for predicting the survival of CC patients. In conclusion, these results suggest that miR-106a is critical for the prognosis outcome of $\mathrm{CC}$ patients. What is more, gain- and loss-of-function experiments demonstrated that miR-106a promoted cell migration, invasion and the expression of invasion-related genes, at least partially by targeting the TIMP2-mediated MMP signaling pathway. Furthermore, miR-106a was inversely correlated with TIMP2 expression, which was downregulated in CC tissues. In addition, miR-106a could negatively modulate TIMP2 accumulation and regulate MMP2 and MMP9 expression, which play an important role in the metastasis of CC. Collectively, these results demonstrated that miR106a functions as oncogene in the migration, invasion and the expression of MMPs by directly inhibiting the TIMP2 pathway.

The degradation of the extracellular matrix (ECM) is crucial for the invasion and metastasis of CC. MMPs, especially MMP2 and MMP9, are able to degrade ECM to promote endothelial cell migration and trigger an angiogenic switch by releasing VEGF from ECM (31). TIMPs are specific inhibitors of MMP2 and MMP9, which were decreased in CC $(32,33)$. Our results revealed that TIMP2 abolished the stimulatory effect of miR-106a on CC cells. Collectively, these data demonstrated that the promotive effect of miR106a was mediated by targeting TIMP2 thus inhibiting MMP expression in CC.

In conclusion, we demonstrated that miR-106a was upregulated in $\mathrm{CC}$ tissues and cell lines, and its increased expression was associated with malignant clinicopathological features. Furthermore, we confirmed that miR-106a promoted cell migration, invasion and the expression of MMPs by inhibiting TIMP2. These results suggest that miR-106a is a potential metastasis-associated tumor oncogene in CC. Collectively, the dysregulation of miR-106a may play an important role in tumor metastasis and may be a novel prognostic factor and potential therapeutic target for CC.

\section{References}

1. Jemal A, Bray F, Center MM, Ferlay J, Ward E and Forman D: Global cancer statistics. CA Cancer J Clin 61: 69-90, 2011.

2. Ferlay J, Shin HR, Bray F, Forman D, Mathers C and Parkin DM: Estimates of worldwide burden of cancer in 2008: GLOBOCAN 2008. Int J Cancer 127: 2893-2917, 2010.

3. Dueñas-Gonzalez A, Cetina L, Mariscal I and de la Garza J: Modern management of locally advanced cervical carcinoma. Cancer Treat Rev 29: 389-399, 2003.

4. Glick SB, Clarke AR, Blanchard A and Whitaker AK: Cervical cancer screening, diagnosis and treatment interventions for racial and ethnic minorities: A systematic review. J Gen Intern Med 27: 1016-1032, 2012.

5. Sakuragi N: Up-to-date management of lymph node metastasis and the role of tailored lymphadenectomy in cervical cancer. Int J Clin Oncol 12: 165-175, 2007.

6. Ambros V: The functions of animal microRNAs. Nature 431: 350-355, 2004.

7. Bartel DP: MicroRNAs: Genomics, biogenesis, mechanism, and function. Cell 116: 281-297, 2004.

8. Su Y,Xiong J,Hu J, Wei X,Zhang X and RaoL: MicroRNA-140-5p targets insulin like growth factor 2 mRNA binding protein 1 (IGF2BP1) to suppress cervical cancer growth and metastasis. Oncotarget 7: 68397-68411, 2016.

9. Yi Y, Li H, Lv Q, Wu K, Zhang W, Zhang J, Zhu D, Liu Q and Zhang W: miR-202 inhibits the progression of human cervical cancer through inhibition of cyclin D1. Oncotarget 7: 72067-72075, 2016.

10. Miller TE, Ghoshal K, Ramaswamy B, Roy S, Datta J, Shapiro CL, Jacob S and Majumder S: MicroRNA-221/222 confers tamoxifen resistance in breast cancer by targeting p $27^{\mathrm{Kip} 1}$. J Biol Chem 283: 29897-29903, 2008.

11. 11. Tsang WP, Ng EK, Ng SS, Jin H, Yu J, Sung JJ and Kwok TT: Oncofetal H19-derived miR-675 regulates tumor suppressor RB in human colorectal cancer. Carcinogenesis 31: 350-358, 2010.

12. Jin Y, Peng D, Shen Y, Xu M, Liang Y, Xiao B and Lu J: MicroRNA-376c inhibits cell proliferation and invasion in osteosarcoma by targeting to transforming growth factor-alpha. DNA Cell Biol 32: 302-309, 2013.

13. Wang Z, Liu M, Zhu H, Zhang W, He S, Hu C, Quan L, Bai J and $\mathrm{Xu}$ N: miR-106a is frequently upregulated in gastric cancer and inhibits the extrinsic apoptotic pathway by targeting FAS. Mol Carcinog 52: 634-646, 2013.

14. Xiao B, Guo J, Miao Y, Jiang Z, Huan R, Zhang Y, Li D and Zhong J: Detection of miR-106a in gastric carcinoma and its clinical significance. Clin Chim Acta 400: 97-102, 2009.

15. Zhu M,Zhang N, He S, Lui Y, Lu G and Zhao L: MicroRNA-106a targets TIMP2 to regulate invasion and metastasis of gastric cancer. FEBS Lett 588: 600-607, 2014.

16. Li P, Xu Q, Zhang D, Li X, Han L, Lei J, Duan W, Ma Q, Wu Z and Wang Z: Upregulated miR-106a plays an oncogenic role in pancreatic cancer. FEBS Lett 588: 705-712, 2014.

17. Wang R, Li Y, Hou Y, Yang Q, Chen S, Wang X, Wang Z, Yang Y, Chen C, Wang Z, et al: The PDGF-D/miR-106a/Twist1 pathway orchestrates epithelial-mesenchymal transition in gemcitabine resistance hepatoma cells. Oncotarget 6: 7000-7010, 2015.

18. Chen L, Zhang F, Sheng XG, Zhang SQ, Chen YT and Liu BW: MicroRNA-106a regulates phosphatase and tensin homologue expression and promotes the proliferation and invasion of ovarian cancer cells. Oncol Rep 36: 2135-2141, 2016.

19. Zhu M, Zhang N, He S, Yan R and Zhang J: MicroRNA-106a functions as an oncogene in human gastric cancer and contributes to proliferation and metastasis in vitro and in vivo. Clin Exp Metastasis 33: 509-519, 2016

20. Hou X, Zhang M and Qiao H: Diagnostic significance of miR-106a in gastric cancer. Int J Clin Exp Pathol 8: 13096-13101, 2015.

21. Yuan R, Zhi Q, Zhao H, Han Y, Gao L, Wang B, Kou Z, Guo Z, He S, Xue X, et al: Upregulated expression of miR-106a by DNA hypomethylation plays an oncogenic role in hepatocellular carcinoma. Tumour Biol 36: 3093-3100, 2015.

22. He QY, Wang GC, Zhang H, Tong DK, Ding C, Liu K, Ji F, Zhu X and Yang S: miR-106a-5p suppresses the proliferation, migration, and invasion of osteosarcoma cells by targeting HMGA2. DNA Cell Biol 35: 506-520, 2016.

23. Zhi F, Zhou G, Shao N, Xia X, Shi Y, Wang Q, Zhang Y, Wang R, Xue L, Wang S, et al: miR-106a-5p inhibits the proliferation and migration of astrocytoma cells and promotes apoptosis by targeting FASTK. PLoS One 8: e72390, 2013. 
24. Ma Y, Zhang H, He X, Song H, Qiang Y, Li Y, Gao J and Wang Z miR-106a inhibits the proliferation of renal carcinoma cells by targeting IRS-2. Tumour Biol 36: 8389-8398, 2015.

25. Yang G, Zhang R, Chen X, Mu Y, Ai J, Shi C, Liu Y, Shi C, Sun L, Rainov NG, et al: miR-106a inhibits glioma cell growth by targeting E2F1 independent of p53 status. J Mol Med (Berl) 89: 1037-1050, 2011.

26. Deng Y, Xiong Y and Liu Y: miR-376c inhibits cervical cancer cell proliferation and invasion by targeting BMI1. Int J Exp Pathol 97: 257-265, 2016.

27. Deng B, Zhang Y, Zhang S, Wen F, Miao Y and Guo K: MicroRNA-142-3p inhibits cell proliferation and invasion of cervical cancer cells by targeting FZD7. Tumour Biol 36 : 8065-8073, 2015.

28. Liu S, Zhang P, Chen Z, Liu M, Li X and Tang H: MicroRNA-7 downregulates XIAP expression to suppress cell growth and promote apoptosis in cervical cancer cells. FEBS Lett 587: 2247-2253, 2013

29. Rothschild SI, Gautschi O, Batliner J, Gugger M, Fey MF and Tschan MP: MicroRNA-106a targets autophagy and enhances sensitivity of lung cancer cells to Src inhibitors. Lung Cancer 107: 73-83, 2017.
30. Servín-González LS, Granados-López AJ and López JA: Families of microRNAs expressed in clusters regulate cell signaling in cervical cancer. Int J Mol Sci 16: 12773-12790, 2015

31. Nagase H and Woessner JF Jr: Matrix metalloproteinases. J Biol Chem 274: 21491-21494, 1999.

32. Cardeal LB, Boccardo E, Termini L, Rabachini T, Andreoli MA, di Loreto C, Longatto Filho A, Villa LL and Maria-Engler SS: HPV16 oncoproteins induce MMPs/RECK-TIMP-2 imbalance in primary keratinocytes: Possible implications in cervical carcinogenesis. PLoS One 7: e33585, 2012.

33. Braicu EI, Fotopoulou C, Chekerov R, Richter R, Blohmer J, Kümmel S, Stamatian F, Yalcinkaya I, Mentze M, Lichtenegger W, et al: Role of serum concentration of VEGFR1 and TIMP2 on clinical outcome in primary cervical cancer: Results of a companion protocol of the randomized, NOGGO-AGO phase III adjuvant trial of simultaneous cisplatin-based radiochemotherapy vs. carboplatin and paclitaxel containing sequential radiotherapy. Cytokine 61: 755-758, 2013 